\title{
The phase function and density of the dust observed at comet 67P/Churyumov-Gerasimenko
}

Marco Fulle, ${ }^{1 \star}$ I. Bertini, ${ }^{2}$ V. Della Corte, ${ }^{3,4}$ C. Güttler, ${ }^{5}$ S. Ivanovski, ${ }^{3,4}$ F. La Forgia, ${ }^{2}$ J. Lasue, ${ }^{6,7}$ A. C. Levasseur-Regourd, ${ }^{8}$ F. Marzari, ${ }^{2}$ F. Moreno, ${ }^{9}$ S. Mottola, ${ }^{10}$ G. Naletto, ${ }^{11,12,13}$ P. Palumbo, ${ }^{4}$ G. Rinaldi, ${ }^{3,4}$ A. Rotundi,,${ }^{3,4}$ H. Sierks, ${ }^{5}$ C. Barbieri, ${ }^{2}$ P. L. Lamy, ${ }^{14}$ R. Rodrigo, ${ }^{15,16}$ D. Koschny, ${ }^{17}$ H. Rickman, ${ }^{18,19}$ M. A. Barucci, ${ }^{20}$ J.-L. Bertaux, ${ }^{21}$ D. Bodewits, ${ }^{22}$ G. Cremonese,${ }^{23}$ V. Da Deppo, ${ }^{13}$ B. Davidsson, ${ }^{24}$ S. Debei, ${ }^{25}$ M. De Cecco, ${ }^{26}$ J. Deller, ${ }^{5}$ S. Fornasier, ${ }^{20}$ O. Groussin, ${ }^{27}$ P. J. Gutiérrez, ${ }^{9}$ H. S. Hviid, ${ }^{10}$ W. H. Ip, ${ }^{28,29}$ L. Jorda, ${ }^{27}$ H. U. Keller, ${ }^{10,30}$ J. Knollenberg, ${ }^{10}$ J. R. Kramm, ${ }^{5}$ E. Kührt, ${ }^{10}$ M. Küppers,${ }^{31}$ M. L. Lara, ${ }^{9}$ M. Lazzarin, ${ }^{2}$ J. J. López-Moreno, ${ }^{9}$ X. Shi, ${ }^{5}$ N. Thomas ${ }^{32,33}$ and C. Tubiana ${ }^{5}$ Affiliations are listed at the end of the paper

Accepted 2018 February 5. Received 2018 January 26; in original form 2017 December 5

\begin{abstract}
The OSIRIS camera onboard Rosetta measured the phase function of both the coma dust and the nucleus. The two functions have a very different slope versus the phase angle. Here, we show that the nucleus phase function should be adopted to convert the brightness to the size of dust particles larger than $2.5 \mathrm{~mm}$ only. This makes the dust bursts observed close to Rosetta by OSIRIS, occurring about every hour, consistent with the fragmentation on impact with Rosetta of parent particles, whose flux agrees with the dust flux observed by GIADA. OSIRIS also measured the antisunward acceleration of the fragments, thus providing the first direct measurement of the solar radiation force acting on the dust fragments and thus of their bulk density, excluding any measurable rocket effect by the ice sublimation from the dust. The obtained particle density distribution has a peak matching the bulk density of most COSIMA particles, and represents a subset of the density distribution measured by GIADA. This implies a bias in the elemental abundances measured by COSIMA, which thus are consistent with the $67 \mathrm{P}$ dust mass fractions inferred by GIADA, i.e. $(38 \pm 8)$ per cent of hydrocarbons versus the $(62 \pm 8)$ per cent of sulphides and silicates.
\end{abstract}

Key words: space vehicles-comets: general-comets: individual: 67P/ChuryumovGerasimenko.

\section{INTRODUCTION}

The OSIRIS Wide-Angle Camera (WAC) onboard Rosetta has observed bursts of dust at distances lower than $100 \mathrm{~m}$ from the spacecraft (Güttler et al. 2017). Three possible scenarios have been proposed to explain the bursts composed of particles of diameters in the range $0.3<d<12 \mathrm{~mm}$ (Güttler et al. 2017). One is the spontaneous ejection of the observed fragments from the dust layer covering Rosetta. COSIMA provides pieces of evidence that such a dust layer is not continuous, and by far thinner than the required thickness of at least $1 \mathrm{~cm}$, needed to eject particles as big as the observed ones (Langevin et al. 2016; Merouane et al. 2017). The second scenario, i.e. the fragmentation of charged fractal particles (Fulle et al. 2015), is excluded by the observed dust accelerations, orders of magnitude lower than expected for optically thin aggregates of sub- $\mu \mathrm{m}$ grains (Mannel et al. 2016). The third possible source of the OSIRIS bursts considers larger parent particles fragmenting on impact with Rosetta, of diameter of about $12 \mathrm{~mm}$ each (Güttler et al. 2017). However, the observed rate of OSIRIS bursts (about one per hour) is completely inconsistent with the measured flux on Rosetta of parent particles of this size. Considering a dust bulk density close to that measured by GIADA, $\rho_{\mathrm{d}}=800 \mathrm{~kg} \mathrm{~m}^{-3}$, Fulle et al. (2016b) find that the production rate of the expected 


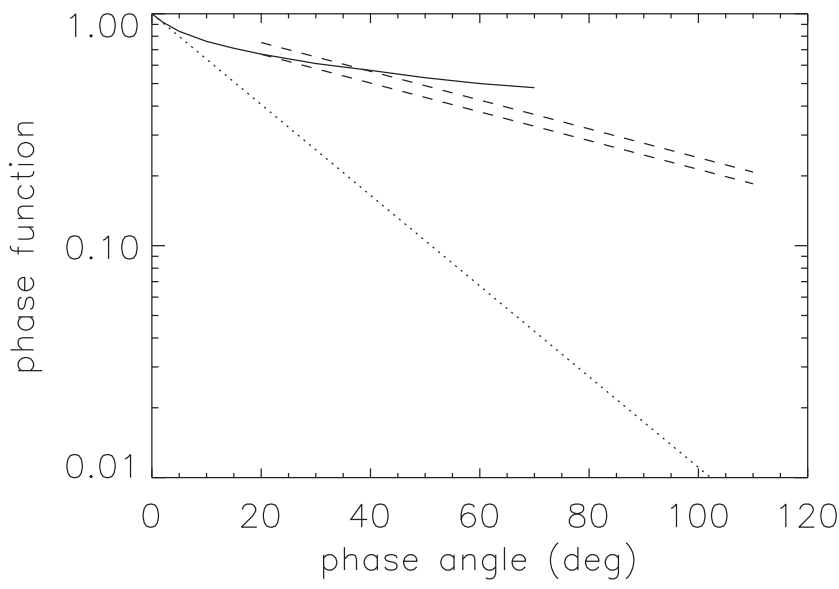

Figure 1. Continuous line: phase function of the Gegenschein dust (Ishiguro et al. 2013). Dotted line: 67P nucleus phase function adopted by Güttler et al. (2017). Dashed lines: average phase function measured in the 67P dust coma on 2015 July and August (Bertini et al. 2017). The upper dashed line is normalized to unity at $\alpha=0^{\circ}$. The lower dashed line is the dust phase function assumed in this paper: it is normalized to the Gegenschein value at $\alpha=20^{\circ}$ and provides the lowest ratio with respect to the nucleus phase function.

$\mathrm{cm}$-sized parent particles ranges from 6 to $12 \times 10^{3} \mathrm{~s}^{-1}$ (see the data in their tables 6 and 8), with a flux at the Rosetta spacecraft between 2.4 and $4.8 \times 10^{-8} \mathrm{~m}^{-2} \mathrm{~s}^{-1}$. This flux, multiplied by the time interval between OSIRIS bursts $(1 \mathrm{~h})$ and the Rosetta cross-section, provides a burst probability ranging from 0.08 to 0.16 per cent, by far inconsistent with the observations. All these facts indicate that the size of the parent particles responsible for the OSIRIS bursts has been largely overestimated. In the next section, we show that a wrong value for the slope of the dust phase function was assumed.

\section{THE DUST ALBEDO TIMES THE PHASE FUNCTION}

For the first time, Bertini et al. (2017) have measured the dust phase function of a comet covering a wide range of phase angles within $2.5 \mathrm{~h}$. This was the shortest time span consistent with the operations of the Rosetta spacecraft, required to sample all phase angles from $0^{\circ}$ to $160^{\circ}$ and to perform the observations. The dust phase function differs significantly from that of the nucleus (Fornasier et al. 2015), which has been adopted to infer the size of individual particles observed in the OSIRIS dust bursts (Güttler et al. 2017) on 2015 June 6 , at phase angles $99<\alpha<111^{\circ}$. The disc-integrated reflectance of the nucleus is well fitted by $A_{\mathrm{n}} \exp \left(-\alpha / \alpha_{\mathrm{n}}\right)$, where $A_{\mathrm{n}}=0.055$ is the nucleus geometric albedo in the orange-filter photometric band at $649.2 \mathrm{~nm}$, and $\alpha_{\mathrm{n}}=22.2$ fixes the phase function slope in the range $0<\alpha<110^{\circ}$ (Güttler et al. 2017). For $20<\alpha<110^{\circ}$, the disc-integrated reflectance of the dust observed on 2015 July and August shows small fluctuations probably due to changes of the dust properties with time and with location in the coma, and is well fitted by $A_{20} \exp \left[\left(\alpha_{20}-\alpha\right) / \alpha_{\mathrm{d}}\right]$, where $A_{20}$ is defined here as the discintegrated reflectance of the dust at the phase angle $\alpha=\alpha_{20}=20^{\circ}$. Bertini et al. (2017) were unable to measure $A_{20}$. The value of $\alpha_{\mathrm{d}}$ is fixed by the dust brightness decrease by the average factor 3.3 from $\alpha=20$ to $110^{\circ}: \alpha_{\mathrm{d}}=70^{\circ}$, much larger than $\alpha_{n}$ (Fig. 1). After 2015 August, the phase function changes a bit its shape (Bertini et al. 2017), probably due to changes in the dust size distribution
(Fulle et al. 2016b). The aim of this section is to check which of these two phase functions provides sizes of the parent particles of the OSIRIS bursts consistent with the dust flux measured at Rosetta by GIADA and OSIRIS. A fit of the dust phase function in terms of the dust size distribution is beyond the scope of this paper.

The cometary analogue best fitting the scattering properties of the particles collected by Rosetta is amorphous carbon (Fulle et al. 2016a), which was assumed to have the light scattering properties of 67P hydrocarbons, the most volume-abundant non-volatile component of 67P (Fulle et al. 2017). Spheres and fractals of hydrocarbons have a geometric albedo $0.05 \leq A_{\mathrm{d}} \leq 0.08$ (Bertini, Thomas \& Barbieri 2007). The link between the dust geometric albedo $A_{\mathrm{d}}$ and $A_{20}$ strongly depends on the dust opposition effect (Poulet et al. 2002; Ishiguro et al. 2013; Dlugach 2016). In order to estimate the link between $A_{\mathrm{d}}$ and $A_{20}$, we consider the phase function of the dust backscattering in the Gegenschein regime (Ishiguro et al. 2013), which is probably a better analogue of cometary dust than the icy grains of Saturn's rings (Poulet et al. 2002; Dlugach 2016). Our choice is consistent with models of the zodiacal cloud, predicting that 90 per cent of its dust has cometary origin (Lasue et al. 2007; Nesvorny et al. 2010; Yang \& Ishiguro 2015). The phase function measured by Ishiguro et al. (2013) in their fig. 10 provides $A_{20} / A_{\mathrm{d}}=0.67$ (Fig. 1), which applied to the geometric albedo of hydrocarbons provides $0.0335 \leq A_{20} \leq 0.0535$. In other words, we assume here that $67 \mathrm{P}$ dust particles have an opposition effect similar to that of the Gegenschein, with a brightness enhancement of about 40 per cent (Ishiguro et al. 2013). Also, Saturn's rings show a similar brightness enhancement due to the opposition effect, but in this case an estimate of $A_{20}$ is impossible because the observations cover a limited phase angle range, namely $0 \leq \alpha \leq 6^{\circ}$ (Poulet et al. 2002).

Since the largest dust coma chunks are pieces of the nucleus surface, there exists a limit chunk size above which $\alpha_{\mathrm{d}}$ becomes $\alpha_{\mathrm{n}}$. From 2015 June to August, the dust particles mainly scattering the visible radiation were smaller than a few tens of $\mu \mathrm{m}$ (Fulle et al. 2016b; Merouane et al. 2017; Moreno et al. 2017), thus explaining why $\alpha_{\mathrm{d}}$ was very different from $\alpha_{\mathrm{n}}$. If the dust limit diameter above which the dust phase function matches the nucleus one is $>0.3 \mathrm{~mm}$, then Güttler et al. (2017) have overestimated the dust cross-section by the factor $A_{20} / A_{\mathrm{n}} \exp \left[\alpha / \alpha_{\mathrm{n}}+\left(\alpha_{20}-\alpha\right) / \alpha_{\mathrm{d}}\right]=25 \pm 10$ for $99<\alpha<111^{\circ}$ and $0.0335 \leq A_{20} \leq 0.0535$. The diameters $d$ of the particles observed close to Rosetta and estimated by Güttler et al. (2017) to cover the range $0.3<d<12 \mathrm{~mm}$ cover instead the range $0.1<d<2.5 \mathrm{~mm}$. Applying the above mentioned factor 25 correction on the cross-section of the OSIRIS bursts parent particles, i.e. decreasing the mass by two orders of magnitude, the production rate of the parent particles increases by three orders of magnitude (Fulle et al. 2016b), making the impact probability larger than one. This shows that only a particular family of dust was the source of the observed OSIRIS dust bursts, and that the dust size correction by a factor $5 \pm 1$ provides a simple and direct explanation of the observations. If the limit dust diameter above which $\alpha_{\mathrm{d}}$ becomes $\alpha_{\mathrm{n}}$ were smaller than $2.5 \mathrm{~mm}$ (i.e. the corrected size of the largest fragments), the largest fragments in the OSIRIS bursts would have the size of $12 \mathrm{~mm}$ provided by the nucleus phase function, and the parent particles as well, because their size mainly depends on that of the largest fragments. Since the flux of parent particles of diameter of $2.5 \mathrm{~mm}$ only is consistent with the OSIRIS bursts rate, we can conclude that the nucleus phase function should not be applied to dust of diameter $d<2.5 \mathrm{~mm}$. The size correction of a factor $5 \pm 1$ to the OSIRIS bursts dust allows us to infer new details of the dust bulk density of 67P dust, as discussed in the next two sections. 


\section{THE DUST BULK DENSITY}

Every dust particle is accelerated antisunward by the solar radiation pressure. For 17 particles with a sufficient number of observations, Güttler et al. (2017) have measured such an acceleration, and have shown that this provides a direct estimate of the dust bulk density assuming a dust spherical shape. Fig. 2 shows the distribution of the dust bulk densities derived from the measured sizes and accelerations, increased by a factor 5 to take into account the same (inverse) size correction. These densities are compared with those derived for the 271 particles of which GIADA measured the mass and crosssection (Colangeli et al. 2007; Della Corte et al. 2014; Fulle et al. 2017), assuming that they had a spherical shape. Fig. 2 shows that the density range of the OSIRIS dust bulk density distribution is completely included in the GIADA one, and that the two distributions have a rather different shape. The densities $\rho_{\mathrm{d}}>5 \times 10^{3} \mathrm{~kg}$ $\mathrm{m}^{-3}$ are unphysical, and actually due to the spherical shape of the particles assumed in Fig. 2 and in the relationship between the dust bulk density and the solar radiation pressure; Fulle et al. (2017) have shown that an aspherical shape with an aspect ratio up to 10 makes the highest densities consistent with cosmochemical data, e.g. silicates and sulphides. On the other side, the lowest densities are due both to the aspherical shape and to the dust porosity (Fulle et al. 2017).

Dust porosity and asphericity make the range of the spherical bulk densities measured by GIADA a bit wider than the range measured by OSIRIS. This fact shows that the dust accelerations measured by OSIRIS are consistent with solar radiation pressure only; no rocket effect due to ice sublimation needs to be invoked to fit the OSIRIS data. This conclusion is in agreement with COSIMA and GIADA observations of dry dust at the spacecraft (Schulz et al. 2015; Fulle et al. 2016a, 2017; Merouane et al. 2016, 2017), and with models of some dust coma features observed by OSIRIS, which suggest a complete ice sublimation within $10 \mathrm{~km}$ from the nucleus surface (Gicquel et al. 2016). The water loss rate observed from Earth-bound satellites around perihelion was never lower than that observed at Rosetta (Shinnaka et al. 2017). This fact evidences that ice sublimation from the 67P dust outside the Rosetta orbiter was always negligible.

The dust bulk density distribution of the OSIRIS dust bursts has a peak matching the bulk density inferred by models of the fragmentation of COSIMA samples on the collection plates (Hornung

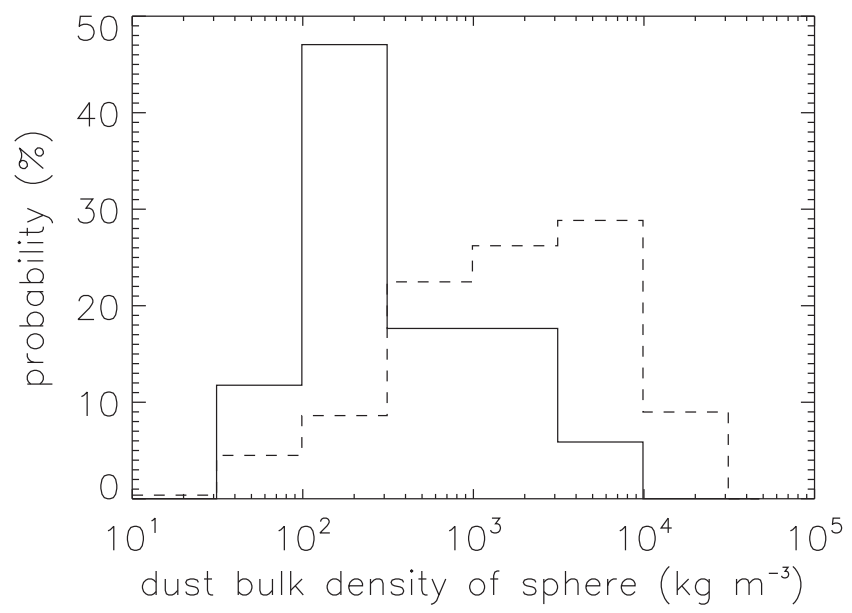

Figure 2. Probability (normalized counts per bin) of the dust bulk density computed assuming a spherical shape. Continuous line: fragments in the OSIRIS bursts. Dashed line: GIADA GDS+IS data (Fulle et al. 2017). et al. 2016). This suggests that the physical properties of the parent particles of both the COSIMA fragments and the OSIRIS bursts are probably the same. COSIMA has also collected denser submm compact particles, probably containing submm crystalline components (Langevin et al. 2017) and thus filling the density bins of $\rho_{\mathrm{d}}>300 \mathrm{~kg} \mathrm{~m}^{-3}$ in Fig. 2.

The fact that the solar radiation pressure fits the dust accelerations observed by OSIRIS definitely excludes the scenario of fragmentation of charged fractal particles, the so-called GIADA showers (Fulle et al. 2015), as a possible source of the OSIRIS bursts. The dust bulk density of the fractal and charged fragments forming the GIADA showers (Fulle et al. 2015) is three orders of magnitude lower than that of the particles forming the OSIRIS bursts, i.e. $<1 \mathrm{~kg} \mathrm{~m}^{-3}$ (Fulle et al. 2015) versus $\approx 800 \mathrm{~kg} \mathrm{~m}^{-3}$. The sizes of the dust fragments in the OSIRIS bursts and in the GIADA showers are similar (Fulle et al. 2015), and the acceleration due to the solar radiation pressure depends inversely on the dust size times the bulk density (Güttler et al. 2017). Therefore, the antisunward acceleration for the charged fractal particles is actually three orders of magnitude larger than the values of $\approx 10^{-4} \mathrm{~m} \mathrm{~s}^{-2}$ obtained by Güttler et al. (2017), i.e. $\approx 0.1 \mathrm{~m} \mathrm{~s}^{-2}$. This is going to push all the charged fragments [decelerated by the spacecraft electric field at speeds $<0.5 \mathrm{~m} \mathrm{~s}^{-1}$ (Fulle et al. 2015)] in the direction of zero phase angle, i.e. out of the OSIRIS-WAC field of view in a very short time. In fact, within the $10 \mathrm{~min}$ of observations of each OSIRIS burst (Güttler et al. 2017), any charged fragments would move $<0.3 \mathrm{~km}$ towards the comet nucleus and about $2 \mathrm{~km}$ in the antisunward direction.

\section{THE RATIO HYDROCARBONS VERSUS MINERALS IN 67P}

On the basis of the results of the Stardust mission (Brownlee 2014), all the dust particles collected by Rosetta can be grouped in three main families, namely sulphides, silicates, and hydrocarbons (Fulle et al. 2016a). Concerning the GIADA particles with measured mass and cross-section (Della Corte et al. 2016a), if we add all the bins in Fig. 2 with $\rho_{\mathrm{d}}>1.5 \times 10^{3} \mathrm{~kg} \mathrm{~m}^{-3}$ (i.e. consistent with the bulk densities of sulphides and silicates), and all the bins with $\rho_{\mathrm{d}}<1.5 \times 10^{3} \mathrm{~kg} \mathrm{~m}^{-3}$ (i.e. consistent with the bulk density of hydrocarbons), then we get similar total probabilities close to 50 percent. Following Stardust samples analysis, which demonstrated that the inhomogeneity of Wild2 material is at the micron scale size both for minerals and for organics (Brownlee et al. 2006; Sandford et al. 2006), it is highly probable that most of $67 \mathrm{P}$ dust particles are mixtures of the three families, so that these mass fractions are necessarily affected by large errors. Better values can be evaluated applying the structural model of the 67P nucleus (Fulle et al. 2016a), which takes into account that the dust particles measured by GIADA are porous mixtures of sulphides, silicates, and hydrocarbons. For a solar $\mathrm{C} / \mathrm{Fe}$ ratio, the structural model of the 67P nucleus provides volume abundances of $(33 \pm 6)$ per cent for sulphides and silicates, and $(67 \pm 6)$ per cent for hydrocarbons (Fulle et al. 2017). Taking into account the bulk density of sulphides, silicates, and hydrocarbons (Fulle et al. 2016a), these volume abundances correspond to mass fractions of $(58 \pm 4)$ per cent for sulphides and silicates versus $(42 \pm 4)$ percent for hydrocarbons. The uncertainty affecting the $\mathrm{C} / \mathrm{Fe}$ ratio of $67 \mathrm{P}$ dust reaches the average between the solar and CI-chondritic values (Bardyn et al. 2017). In this case, the structural model of the 67P nucleus provides volume abundances of $(42 \pm 6)$ per cent for sulphides and silicates, and ( $58 \pm 6$ ) per cent for hydrocarbons (Fulle et al. 2016a), 
corresponding to mass fractions of $(67 \pm 4)$ percent for sulphides and silicates versus $(33 \pm 4)$ percent for hydrocarbons. We can conclude that GIADA observes average mass fractions of $(62 \pm 8)$ per cent for sulphides and silicates versus $(38 \pm 8)$ per cent for hydrocarbons.

The GIADA dust bulk densities shown in Fig. 2 were actually extracted from a subset only of the collected particles, i.e. those detected by both the GDS and IS subsystem (GDS+IS particles), providing the dust cross-section, mass, and velocity (Della Corte et al. 2015, 2016b). GDS (Grain Detection System) measures the light scattered by the particles crossing a laser curtain at GIADA entrance. IS (Impact Sensor) is a piezoelectric sensor placed below GDS and measuring the momentum transferred by the impacting particles. GIADA detected other three subsets of particles: IS only detections, single GDS only detections, and GDS detections in showers (Fulle et al. 2015, 2016a), whose contribution can be neglected in this analysis. In fact, the GDS showers were modelled in terms of fragmentation of fractal parent particles, and have a mass so low to provide a negligible contribution to the overall composition of the 67P dust (Fulle et al. 2015). IS only detections are compact and dense particles which are too small to be detected by GDS and would fall on the right-hand bins of Fig. 2. GDS single detections are too porous to transfer any momentum to the IS sensor and would fall on the left-hand bins of Fig. 2. Thus, even though the density distribution provided by GIADA (Fig. 2) is obtained using GDS+IS detections only, this does not introduce any significant bias, being the other two subsets of detections similar in number (Fulle et al. 2015) and contributing to the right part and to the left part of the diagram, respectively.

The OSIRIS distribution of the dust bulk densities is instead affected by a significant bias. It also samples all the three groups observed by GIADA, i.e. sulphides, silicates, and hydrocarbons, as clearly shown by the filled density bins (Fig. 2). However, the peak and shape of the OSIRIS density distribution are very far from the GIADA one. The explanation is evident: only the parent particles porous enough to fragment can be the source of the OSIRIS bursts. Particles as compact as those observed by GIADA in the density bins of $\rho_{\mathrm{d}}>3 \times 10^{3} \mathrm{~kg} \mathrm{~m}^{-3}$, or by Stardust as carrot-like type-A tracks with a single terminal particle (Burchell et al. 2008) are too strong to fragment on impact with Rosetta. They either stick or bounce with a negligible probability to be observed by OSIRIS at the rate of a single out-of-focus particle per hour. Most COSIMA particles have the same bulk density as the OSIRIS density peak in Fig. 2 (Hornung et al. 2016), and thus probably a similar density distribution, being fragments of parent particles of similar physical properties. This implies a composition bias affecting the elemental abundances of the particles belonging to this group only: the OSIRIS fragments are both more porous and richer in hydrocarbons with respect to silicates and sulphides, when compared to the complete GIADA set. This is consistent with the mass fraction of hydrocarbons measured by COSIMA, ( $45 \pm 15$ ) per cent (Bardyn et al. 2017), with an average value slightly larger than the $(38 \pm 8)$ per cent inferred by GIADA. The sampling bias affecting the COSIMA data will prevent to significantly reduce the uncertainty of the $67 \mathrm{P}$ dust composition.

\section{CONCLUSIONS}

The OSIRIS dust bursts have only one plausible explanation, namely the fragmentation of larger parent particles impacting Rosetta. The flux of these particles has been determined by the observations of single particles around Rosetta (Rotundi et al. 2015; Fulle et al. 2016b; Ott et al. 2017) and provides strong constraints on the possible rate of the OSIRIS bursts. This consideration allows us to conclude that the dust phase function measured by OSIRIS (Bertini et al. 2017) should be applied to dust diameters up to $2.5 \mathrm{~mm}$ at least in order to convert dust brightness into dust size. There exists surely a chunk size above which the dust phase function matches the nucleus phase function, but models able to infer this precise threshold are currently unavailable. It is improbable that this threshold is larger than the largest chunk sizes observed in the 67P coma, so that now it is impossible to infer any correction of the flux of the $67 \mathrm{P}$ chunks, which has been evaluated adopting the nucleus phase function (Fulle et al. 2016b; Ott et al. 2017). What we find also has implications on the structure of the nucleus surface: the smallest size unit that replicates the surface structure has to be $>2.5 \mathrm{~mm}$.

The antisunward dust acceleration measured in the OSIRIS bursts is perfectly consistent with solar radiation pressure, and allows us to exclude any significant ice sublimation from the dust fragments and to infer the dust bulk density of the fragments. This is orders of magnitude larger than the bulk density of one MIDAS fractal particle (Mannel et al. 2016) and of the fragments observed in the GIADA showers (Fulle et al. 2015). These fractal particles, deflected by the spacecraft electric field at very low velocities, are pushed by the solar radiation pressure in the antisunward direction in a few seconds, thus never entering the field of view of the OSIRIS and navigation cameras during most observations, pointing at the nucleus from the terminator. The bulk density distribution of the OSIRIS dust bursts is a subset of that measured by GIADA (Fulle et al. 2017), and is composed only of the particles porous enough to fragment on impact with Rosetta. It has a peak matching the bulk density inferred in most COSIMA samples fragmented on the collection plates (Hornung et al. 2016), suggesting a similar bulk density distribution for most COSIMA samples too. This implies a bias in the elemental abundances measured by COSIMA, which thus are consistent with the 67P dust mass fractions inferred by GIADA, i.e. $(38 \pm 8)$ per cent of hydrocarbons, versus the $(62 \pm 8)$ per cent of sulphides and silicates.

\section{ACKNOWLEDGEMENTS}

We thank the anonymous referee for having significantly improved the paper; and the Rosetta Science Ground Segment at ESAC, the Rosetta Mission Operations Centre at ESOC, and the Rosetta Project at ESTEC for their outstanding work enabling the science return of the Rosetta Mission. All GIADA data presented here are available on request before archiving in the PSA. This research was supported by the Italian Space Agency (ASI) within the ASI-INAF agreements $\mathrm{I} / 032 / 05 / 0$ and $\mathrm{I} / 024 / 12 / 0$.

\section{REFERENCES}

Bardyn A. et al., 2017, MNRAS, 469, S712

Bertini I., Thomas N., Barbieri C., 2007, A\&A, 461, 351

Bertini I. et al., 2017, MNRAS, 469, S404

Brownlee D. E., 2014, Annu. Rev. Earth Planet. Sci., 42, 179

Brownlee D. E. et al., 2006, Science, 314, 1711

Burchell M. J. et al., 2008, Meteorit. Planet. Sci., 43, 23

Colangeli L. et al., 2007, Adv. Space Res., 39, 446

Della Corte V. et al., 2014, Int. J. Astron. Instr., 3, 1350011

Della Corte V. et al., 2015, A\&A, 583, A13

Della Corte V. et al., 2016a, Acta Astron., 126, 205

Della Corte V. et al., 2016b, MNRAS, 462, S210

Dlugach J. M., 2016, J. Quant. Spectrosc. Radiat. Transfer, 183, 38

Fornasier S. et al., 2015, A\&A, 583, A30 
Fulle M. et al., 2015, ApJ, 802, L12

Fulle M. et al., 2016a, MNRAS, 462, S132

Fulle M. et al., 2016b, ApJ, 821, 19

Fulle M. et al., 2017, MNRAS, 469, S45

Gicquel A. et al., 2016, MNRAS, 462, S57

Güttler C. et al., 2017, MNRAS, 469, 312

Hornung K. et al., 2016, Planet. Space Sci., 133, 63

Ishiguro M., Yang H., Usui F., Pyo J., Ueno M., Ootsubo T., Minn Kwon S., Mukai T., 2013, ApJ, 767, 75

Langevin Y. et al., 2016, Icarus, 271, 76

Langevin Y. et al., 2017, MNRAS, 469, S535

Lasue J., Levasseur-Regourd A. C., Fray N., Cottin H., 2007, A\&A, 473, 641

Mannel T., Bentley M. S., Schmied R., Jeszenszky H., Levasseur-Regourd A. C., Romstedt J., Torkar K., 2016, MNRAS, 462, S304

Merouane S. et al., 2016, A\&A, 596, A87

Merouane S. et al., 2017, MNRAS, 469, S459

Moreno F. et al., 2017, MNRAS, 469, S186

Nesvorny D., Jenniskens P., Levison H. F., Bottke W. F., Vokrouhlický D., Gounelle M., 2010, ApJ, 713, 816

Ott T. et al., 2017, MNRAS, 469, S276

Poulet F., Cuzzi J. N., French R. G., Dones L., 2002, Icarus, 158, 224

Rotundi A. et al., 2015, Science, 347, aaa3905

Sandford S. A. et al., 2006, Science, 314, 1720

Schulz R. et al., 2015, Nature, 518, 216

Shinnaka Y. et al., 2017, AJ, 153, 76

Yang H., Ishiguro M., 2015, ApJ, 813, 87

${ }^{1}$ INAF - Osservatorio Astronomico, Via Tiepolo 11, I-34143 Trieste, Italy

${ }^{2}$ Department of Physics and Astronomy 'G. Galilei', University of Padova, Vic. Osservatorio 3, I-35122 Padova, Italy

${ }^{3}$ INAF - Istituto di Astrofisica e Planetologia Spaziali, Via Fosso del Cavaliere, 100, I-00133 Rome, Italy

${ }^{4}$ Universitá degli Studi di Napoli Parthenope, Dip. di Scienze e Tecnologie, CDN IC4, I-80143 Naples, Italy

${ }^{5}$ Max-Planck-Institut für Sonnensystemforschung, Justus-von-Liebig-Weg, 3, D-37077 Göttingen, Germany

${ }^{6}$ University of Tolouse, UPS-OMP, IRAP, F-31400 Toulouse, France

${ }^{7}$ CNRS, IRAP, 9 avenue colonel Roche, BP 44346, F-31028 Toulouse Cedex 4, France

${ }^{8}$ Sorbonne Université; UVSQ (UPSay); CNRS/INSU; LATMOS-IPSL, BC 102, Campus UPMC, 4 place Jussieu, F-75005 Paris, France

${ }^{9}$ Instituto de Astrofisica de Andalucia CSIC, Glorieta de la Astronomia, E-18008 Granada, Spain

${ }^{10}$ Deutsches Zentrum für Luft- und Raumfahrt (DLR), Institut für Planetenforschung, Rutherfordstrasse 2, D-12489 Berlin, Germany

${ }^{11}$ Department of Physics and Astronomy 'G. Galilei', University of Padova, Via Marzolo 8, I-35131 Padova, Italy
${ }^{12}$ Center of Studies and Activities for Space, CISAS, 'G. Colombo', University of Padova, Via Venezia 15, I-35131 Padova, Italy

${ }^{13}$ CNR-IFN UOS Padova LUXOR, Via Trasea 7, I-35131 Padova, Italy

${ }^{14}$ Laboratoire d'Astrophysique de Marseille, UMR 7326, CNRS and Aix Marseille Université, F-13388 Marseille Cedex 13, France

${ }^{15}$ Centro de Astrobiologia, CSIC-INTA, E-28850 Torrejon de Ardoz, Madrid, Spain

${ }^{16}$ International Space Science Institute, Hallerstrasse 6, CH-3012 Bern, Switzerland

${ }^{17}$ Science Support Office, European Space Research and Technology Centre/ESA, Keplerlaan 1, Postbus 299, NL-2201 AZ Noordwijk ZH, the Netherlands

${ }^{18}$ Department of Physics and Astronomy, Uppsala University, Box 516, SE75120 Uppsala, Sweden

${ }^{19}$ Space Research Center, Bartycka 18A, PL-00716 Warszawa, Poland

${ }^{20}$ Observatoire de Paris, PSL Research University, CNRS, Univ. Paris

Diderot, Sorbonne Paris Cité, UPMC Univ. Paris 06, Sorbonne Universités, 5 place Jules Janssen, F-92195 Meudon, France

${ }^{21}$ LATMOS, CNRS/UVSQ/IPSL, 11 Boulevard d'Alembert, F-78280 Guyancourt, France

${ }^{22}$ Department of Astronomy, University of Maryland, College Park, MD 20742-2421, USA

${ }^{23}$ INAF, Osservatorio Astronomico di Padova, Vicolo dell'Osservatorio 5, I-35122 Padova, Italy

${ }^{24}$ Jet Propulsion Laboratory, M/S 183-301, 4800 Oak Grove Drive, Pasadena, CA 91109, USA

${ }^{25}$ Department of Industrial Engineering, University of Padova, Via Venezia 1, I-35131 Padova, Italy

${ }^{26}$ Faculty of Engineering, University of Trento, via Mesiano 77, I-38121 Trento, Italy

${ }^{27}$ Aix Marseille Université, CNRS, LAM (Laboratoire d'Astrophysique de Marseille) UMR 7326, F-13388 Marseille, France

${ }^{28}$ Graduate Institute of Astronomy, National Central University, 300 ChungDa Rd, Chung-Li 32054, Taiwan

${ }^{29}$ Space Science Institute, Macau University of Science and Technology, Avenida Wai Long, Taipa, Macau

${ }^{30}$ Institut für Geophysik und extraterrestrische Physik, Technische Universität Braunschweig, Mendelssohnstr. 3, D-38106 Braunschweig, Germany

${ }^{31}$ Operations Department, European Space Astronomy Centre/ESA, PO Box 78, E-28691 Villanueva de la Cañada (Madrid), Spain

${ }^{32}$ Physikalisches Institut der Universität Bern, Sidlerstr. 5, CH-3012 Bern, Switzerland

${ }^{33}$ Center for Space and Habitability, University of Bern, CH-3012 Bern, Switzerland

This paper has been typeset from a $\mathrm{T}_{\mathrm{E}} \mathrm{X} / \mathrm{L} \mathrm{T} \mathrm{E} \mathrm{X}$ file prepared by the author. 